\section{Reproductive and genetic consequences of founding isolated lion populations}

\author{
D. E. Wildt, M. Bush, K. L. Goodrowe, C. Packer*, \\ A. E. Pusey*, J. L. Brown $†$, P. Joslin $\ddagger$ \& S. J. O’Brien§
}

National Zoological Park, Smithsonian Institution, Washington, District of Columbia 20008, USA

* Department of Ecology and Behavioral Biology, University of Minnesota, Minneapolis, Minnesota 55455, USA

$\dagger$ Department of Obstetrics and Gynecology, Uniformed Services University of the Health Sciences, Bethesda, Maryland 20814, USA $\ddagger$ Chicago Zoological Park, Brookfield, Illinois 60513, USA

$\S$ Section of Genetics, Laboratory of Viral Carcinogenesis, National Cancer Institute, Frederick, Maryland 21701, USA

Species survival is critically dependent on reproductive performance, a complex physiological process under rigorous genetic control. Classical studies of inbreeding in laboratory animals and livestock have shown that increased homozygosity can adversely affect spermatogenesis, ovulation and perinatal mortality and morbidity $^{1-3}$. For wild populations, the consequences of inbreeding depression have not been examined intensively, although our recent studies of the African cheetah revealed a striking degree of genetic uniformity ${ }^{4,5}$ combined with an extremely high incidence of structurally abnormal spermatozoa $(>70 \%)$ in captive ${ }^{6}$ as well as free-ranging ${ }^{7}$ males. In this study, we report definitive evidence that the reproductive function of free-ranging mammals can be impaired as a result of demographic contraction followed by inbreeding. In an examination of three distinct lion populations (two from the Serengeti ecosystem in East Africa and a third descended from lions in the Gir Forest of western India), a direct correlation was observed between genetic variability and two physiological traits, incidence of abnormal sperm and circulating testosterone, a critical hormone for spermatogenesis.

The lion (Panthera leo) is an attractive candidate for studying the interrelationship of genetics and reproduction. Lions have been studied extensively for centuries, and their numbers in Africa are vast (estimated as high as 200,000$)^{8}$. The demography and behaviour of the lions in the Serengeti ecosystem ( P. l. leo) and the Gir Forest Sanctuary ( $P$. l. persica) have been monitored for 20 years or more e-15 $^{9}$

The lion is the most social of all the Felidae. Pride composition may remain more or less stable for several years and is affected primarily by birth, death, emigration of subadults and takeovers by non-resident coalitions of males ${ }^{12,13}$. The pride core is a closed society of lionesses, all genetically interrelated. Inbreeding is normally avoided because, with rare exceptions, young males abandon the pride by 3.5 years of age, reducing the potential for incestuous matings ${ }^{13}$. Additionally, adult lionesses solicit matings from nomadic males or males from neighbouring prides, making inbreeding extremely infrequent. A possible exception is in severely restricted habitats, where a lack of unrelated partners inevitably results in incestuous sexual encounters.

Three distinct lion populations were surveyed, two in the Serengeti ecosystem in Tanzania, East Africa: one in the Serengeti National Park and the second in the Ngorongoro Crater. Approximately 3,000 lions inhabit the Serengeti Plains, an area of $>25,000 \mathrm{~km}^{2}$. The Ngorongoro Crater is a large volcanic caldera in the southeastern portion of the Serengeti ecosystem containing one of the highest lion population densities (100 animals in $260 \mathrm{~km}^{2}$ ) in Africa ${ }^{13}$. Plentiful prey biomass and a suboptimal habitat immediately outside the Crater provide little incentive for lions to leave the area. Occasionally, resident lions emigrate but no immigration into the Crater has been recorded since 1975 when continuous identification of individual lions began ${ }^{13}$. Thus, the Ngorongoro Crater is essentially a selfcontained ecological niche containing a distinct and isolated population of lions. The present population is known to have descended from a small number $(6-15)$ of founders which survived a 1962 epizootic of biting flies (Stomoxys calcitrans) ${ }^{16}$. The population gradually increased to its present size in 1975 and has remained stable since ${ }^{12,13}$. The third group surveyed originated from lion stock of the Gir Forest Sanctuary located in the Gujarat Province of western India. These Asian lions, which show several morphological distinctions from their African counterparts ${ }^{14,15}$, are a relict group of $<250$ individuals which experienced a severe population contraction (to $<20$ animals) in the first quarter of this century ${ }^{17}$. The lions sampled were in captivity at the Sakkarbaug Zoo near the Gir Forest.

In parallel studies, blood samples from lions of each popula-
Fig. 1 Pleiomorphic sperm forms in the ejaculates of lions. Males were anaesthetized with Telazol (Tiletamine plus Zolazepam, Warner Lambert, $3 \mathrm{mg}$ per $\mathrm{kg}$ ) administered with a dart gun. Semen was obtained by a standardized electroejaculation procedure ${ }^{6,7}$. After fixing seminal aliquots from each animal in $1 \%$ glutaraldehyde, the morphologies of 900 individual spermatozoa from each male were assessed by phase contrast microscopy $(\times 400)$. $a$, Normal; $b$, tightly coiled flagellum; $c$, missing mitochondrial sheath; $d$, abnormal acrosome and deranged midpiece; $e$, macrocephalic with abnormal acrosome; $f$, microcephalic with missing mitochondrial sheath; $g$, bent flagellum; $h$, bent neck with residual cytoplasmic droplet.
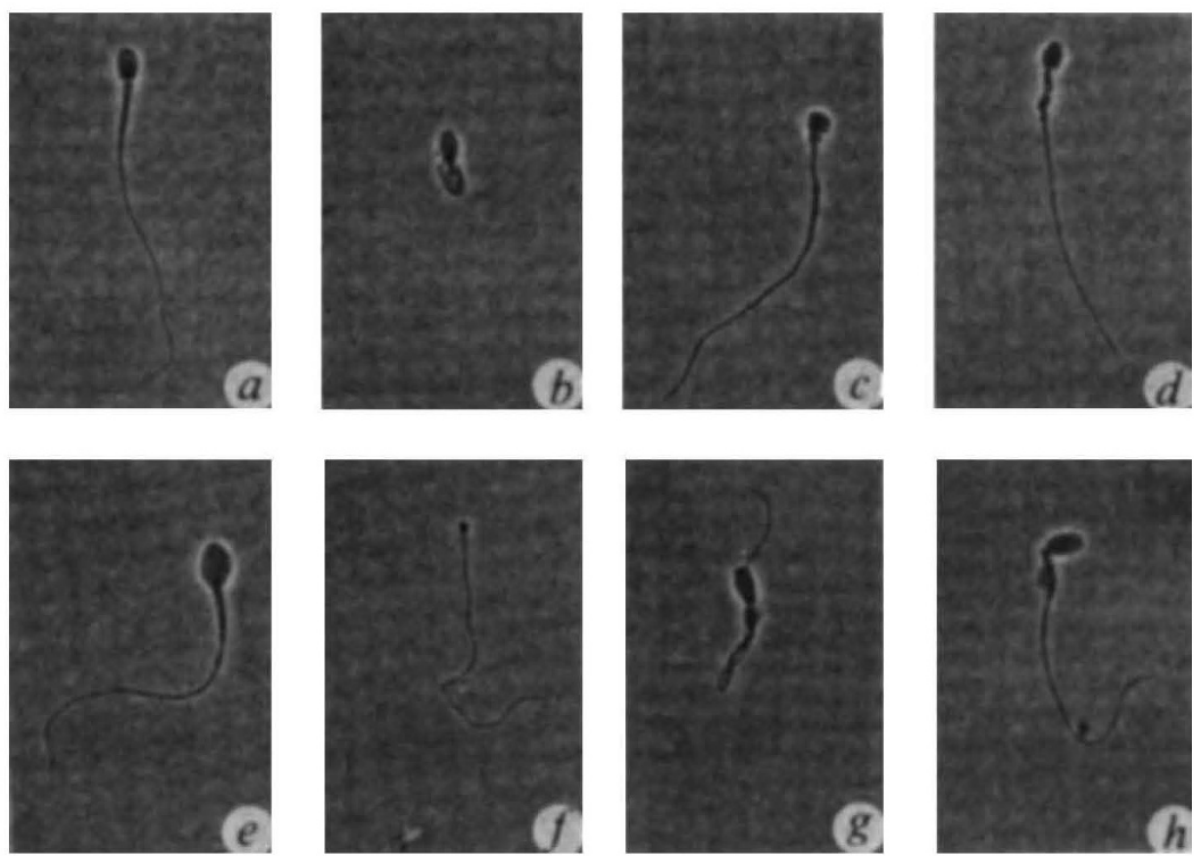
Table 1 Ejaculate characteristics of lions from the Serengeti Plains, the Ngorongoro Crater and the Sakkarbaug Zoo

\begin{tabular}{|c|c|c|c|}
\hline Lions tested & $\begin{array}{c}\text { Serengeti } \\
\text { National Park } \\
n=8\end{array}$ & $\begin{array}{c}\text { Ngorongoro } \\
\text { Crater } \\
n=9\end{array}$ & $\begin{array}{c}\text { Sakkarbaug } \\
\text { Zoo } \\
n=8\end{array}$ \\
\hline Ejaculate volume (ml) & $9.4 \pm 1.4^{\mathrm{a}}$ & $8.5 \pm 0.8^{\mathrm{a}}$ & $5.9 \pm 0.7^{\mathrm{b}}$ \\
\hline Spermatozoal motility (\%) & $91.0 \pm 4.2^{\mathrm{a}}$ & $83.0 \pm 4.6^{\mathrm{a}}$ & $61.0 \pm 3.7^{\mathrm{b}}$ \\
\hline Motile sperm per ejaculate $\left(\times 10^{6}\right)$ & $228.5 \pm 65.5^{\mathrm{a}}$ & $236.0 \pm 93.0^{\mathrm{a}}$ & $45.3 \pm 9.9^{\mathrm{b}}$ \\
\hline Total sperm abnormalities (\%) & $24.8 \pm 4.0^{\mathrm{a}}$ & $50.5 \pm 6.8^{\mathrm{b}}$ & $66.2 \pm 3.6^{\mathrm{b}}$ \\
\hline \multicolumn{4}{|l|}{ Type: } \\
\hline (3) Biflagellate & $0.04 \pm 0.03$ & $0.03 \pm 0.02$ & $0.0 \pm 0.0$ \\
\hline (4) Bicephalic & $0.2 \pm 0.04$ & $0.8 \pm 0.6$ & $0.4 \pm 0.2$ \\
\hline (5) Abnormal acrosome & $1.1 \pm 0.3^{\mathrm{a}}$ & $0.9 \pm 0.1^{\mathrm{a}}$ & $3.6 \pm 0.7^{b}$ \\
\hline (6) Abnormal midpiece & $1.9 \pm 0.4^{\mathrm{a}}$ & $3.7 \pm 1.0^{\mathrm{a}}$ & $0.6 \pm 0.2^{\mathrm{b}}$ \\
\hline (7) Tightly coiled flagellum & $2.3 \pm 0.5^{\mathrm{a}}$ & $8.5 \pm 3.3^{\mathrm{b}}$ & $13.7 \pm 2.4^{\mathrm{c}}$ \\
\hline (8) Detached head & $0.0 \pm 0.0^{\mathrm{a}}$ & $0.0 \pm 0.0^{\mathrm{a}}$ & $6.6 \pm 1.8^{\mathrm{b}}$ \\
\hline (9) Bent midpiece with droplet & $2.3 \pm 0.6^{\mathrm{a}}$ & $12.4 \pm 3.0^{\mathrm{b}}$ & $5.2 \pm 1.0^{\mathrm{c}}$ \\
\hline
\end{tabular}

Each ejaculate was collected in a warmed $\left(37^{\circ} \mathrm{C}\right)$ plastic container. Sperm motility and concentration values were evaluated immediately in the field ${ }^{6,7}$ using a phase-contrast microscope powered by a portable generator. These values as well as semen volume were used to provide the index of motile sperm per ejaculate. Fig. 1 legend describes general methodology for morphological assessments. Within each row, values with different superscripts are significantly different $(P<0.05)$; values within rows with no superscripts are $\operatorname{similar}(P>0.05)$.

tion were separated into erythrocytes, leucocytes and plasma for preparation of isozyme extracts ${ }^{18}$. An electrophoretic survey of 46-50 allozyme loci revealed measurable differences in the extent of genetic variation among the three populations. The Serengeti group $(n=27)$ had abundant genetic variation with the proportion of the loci polymorphic, $P=10.9 \%$. The mean heterozygosity $(H$, the average frequency of heterozygous loci in one individual) was 0.038 . The Crater lions $(n=19)$ expressed less variability $(P=4.3 \% ; H=0.014)$, and there was allelic variation at a subset of the loci that were polymorphic in the surrounding (and presumably founder) population of the Serengeti Plains. The Asiatic lion samples $(n=28)$ from the Sakkarbaug Zoo were unique among the three groups in demonstrating complete genetic monomorphism at each of the typed loci.

To quantify physiological differences among lions from the three environments, seminal and endocrine traits were evaluated and related to genetic findings. Semen samples were collected from anaesthetized lions in July and August in Africa and November in India using a standardized electroejaculation procedure $^{6,7}$. Based on previous observational studies, the precise age of each African animal was known and none of the males had been observed copulating or consorting with oestrous females during the 6 weeks before semen collection. The age (Serengeti, $5.3 \pm 0.8$ years; Crater, $4.5 \pm 0.5$ years; mean \pm s.e.m.) and age range (Serengeti, 2.5-7.0 years; Crater, 3.0-7.5 years) of each African g. Jup conformed to sexual maturity, onset of spermatogenesis normally occurring at 24-36 months (ref. 9). An accurate average age could not be calculated for the Asian lions because one was a founder of unknown age caught in the wild; however, the age range of the captive-born descendents (4.5-9.0 years) was similar to that of the African groups. Before, during and after the electroejaculation interval, blood samples were collected and sera were stored and later analysed for luteinizing hormone ( $\mathrm{LH})$ and testosterone concentrations.

Compared to African lions, the Asiatic lions showed depressed mean ejaculate volume, a sperm motility rating and fewer motile sperm per ejaculate (Table 1). Although the two African populations were comparable in these traits, $50.5 \%$ of the spermatozoa from the Crater lions were morphologically abnormal, a level twofold greater than that detected in the Serengeti lions (Table 1). Lions at the Sakkarbaug Zoo averaged
$16 \%$ more pleiomorphic spermatozoa than the Crater group and a threefold greater incidence of morphological abnormalities than observed in the Serengeti population (Table 1). The greatest increases in structural defects were observed in the number of spermatozoa with a tightly coiled flagellum or deranged midpiece (Table 1; Fig. 1). Between the Crater and Sakkarbaug Zoo groups, the specific proportion of abnormalities frequently varied, with the former producing more sperm with midpiece defects whereas sperm from the latter generally were affected with more acrosomal and flagellar deformities. Certain Crater and Sakkarbaug Zoo lions were predisposed to a high incidence of very unusual abnormalities. For example, $5.4 \%$ of all sperm cells in one Crater male were afflicted by bi- or tricephaly whereas in two Asian males, $12.4 \%$ and $15.4 \%$ of all sperm heads were decapitated from the flagellar portion of the cell. The precise aetiology of specific pleiomorphic forms is controversial $^{19}$. However, defect types 1-8 (Table 1) are generally associated with spermatogenic dysfunction whereas type 9-13 defects originate as sperm migrate through the excurrent duct system $^{6,7}$. In either case, the fertilization potential of an abnormal spermatozoon is probably impaired because of severely reduced cell motility, acrosomal integrity and perhaps even altered DNA content in instances of micro- or macrocephaly ${ }^{20}$.

LH is an important gonadotropin in the male and is primarily responsible for stimulating testosterone production by the Leydig cells. As measured eight times over an $\sim 80$ min interval, the LH profile was similar in the three populations (Fig. 2). Mean testosterone concentrations, however, were about threefold less $(P<0.05)$ in the Crater and Sakkarbaug Zoo populations compared to the Serengeti group (Fig. 2). The overall temporal testosterone profile was not different $(P>0.05)$ in Crater compared to Sakkarbaug Zoo lions; however, as the blood sampling interval progressed beyond $20 \mathrm{~min}$, testosterone concentrations were two- to fourfold less in Sakkarbaug Zoo compared to Crater lions.

The integration of pituitary and gonadal hormones is essential for normal spermatogenesis ${ }^{21,22}$. African lions in the restricted Ngorongoro habitat or descended from the remnant Gir Forest population had lower testosterone levels even when basal concentrations of the controlling hormone $\mathrm{LH}$ were equivalent. Reduced testosterone secretion can contribute to impaired 
spermatogenesis by interfering with meiotic events associated with normal gamete formation ${ }^{23,24}$. The physiological basis for lowered testosterone production is unclear, but could involve impaired hypothalamic, pituitary or testicular function.

Overall, these results can be interpreted in one of two ways. It is possible that the differences among populations (particularly between the African and Asian groups) was, in part, attributable to variations in environmental factors (seasonality, nutrition, pride dynamics, copulatory activity, or whether the lions were free-ranging or captive-bred). These variables were difficult to control in the present study but nonetheless were minimized as a result of our previous efforts involving longitudinal ethological observations of both African and Asian populations. Environmental variability is an unlikely explanation of the data for several reasons. First, lion reproductive performance is not markedly seasonal ${ }^{9}$ and the times of semen evaluation corresponded to previous field observations of breeding activity in each population ${ }^{12-15}$. Secondly, although the Serengeti and Ngorongoro Crater populations are strikingly distinct in genetic and reproductive correlates, these lions reside in adjacent ecosystems involving the same climate, pride structure and prey base. Further, these two groups, sampled at the same time of year, were age-matched and precisely selected on the basis of inter- and intra-pride reproductive experience. Finally, comparing reproductive characteristics between the free-living African and captive Asian populations seems justifiable because there is at least one precedent for male reproductive traits not varying between captive and free-living felids; there was no difference in semen characteristics and circulating testosterone levels between cheetahs maintained in various US zoological parks and those free-ranging in the Serengeti ecosystem ${ }^{7}$.

A more logical explanation of the results is that as allozyme variation decreased among geographical groups, ejaculate quality declined, with sperm structure appearing most vulnerable. Spermatozoal morphology is not influenced by ejaculatory abstinence in a variety of felids (including domestic cats, cheetahs ${ }^{6}$ and clouded leopards ${ }^{25}$ ) but rather remains consistent even among seminal aliquots collected as frequently as three times per week. Additionally, the semen characteristics measured in this study were based on a highly standardized electroejaculation protocol (all males received the same number and type of stimuli under the same level of surgical anaesthesia) previously shown to be effective in discriminating among male felids with variable reproductive performance records ${ }^{25}$. Furthermore, not only was there an inverse correlation between genetic variability and spermatozoal integrity, but there was a correlative and significant depression in circulating testosterone in both the lion populations with lower quality spermatozoa. Taken together, these results implicate the genetic history of the populations in contributing to reproductive status in a manner reminiscent of reproductive impairment in deliberately inbred mice and farm livestock ${ }^{1-3}$. The data from the two African lion populations suggest that a restricted natural habitat combined with a well-defined population bottleneck affected genotype and spermatozoal integrity. The Asian lion data strongly support the hypothesis that diminished genotypic diversity adversely influences seminal traits as these lions produce ejaculates with more pleiomorphic sperm forms and fewer motile spermatozoa than even the reproductively compromised Crater lions.

Overall reproductive success in lions could be influenced by altered degrees of spermatogenesis and testosterone-influenced sexual activity in concert with normal inter- and intra-pride dynamics. The ultimate effect of impaired reproductive function in the Crater lions may not be recognized for years, if ever. However, recent breeding performance of some of the Sakkarbaug Zoo lions may warrant concern. One of the 6.5-year-old test males mated to oestrous females has failed to produce offspring and three of the other males have produced only single-cub litters or stillborn cubs in the past two years. Also of
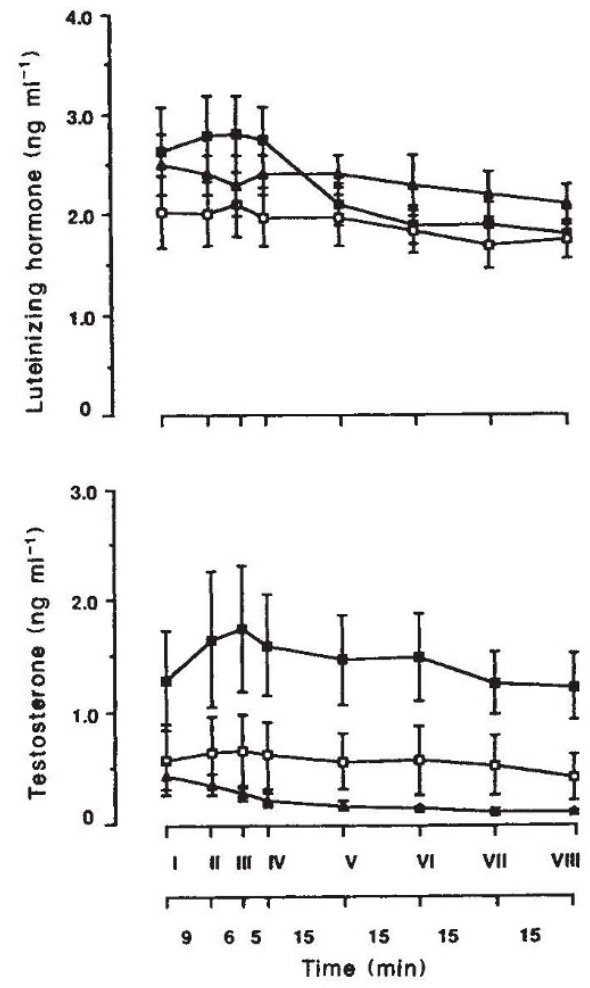

Fig. 2 Mean ( \pm s.e.m.) concentrations of serum LH and testosterone in lions from the Serengeti National Park ( $\square$ ), the Ngorongoro Crater $(\square)$ and the Sakkarbaug Zoo (A). Roman numerals indicate time of blood sampling: I, post-anaesthesia, pre-electroejaculation; II-IV, end of electroejaculation series 1, 2 and 3, respectively; V-VIII, 15, 30, 45 and 60 min after electroejaculation. Serum samples were analysed using validated radioimmunoassays ${ }^{7,25,27,28}$. A biomedical computer program (Statistical Analysis System Institute) was used to evaluate differences in repeated measures of $\mathrm{LH}$ and testosterone.

significance is the potential influence of a homozygous genotype on the health status of the species, as illustrated in the devastating susceptibility of the cheetah to a corona virus, feline infectious peritonitis ${ }^{26}$.

Together, these results demonstrate the conceivable impact of a known population bottleneck on physiological function of a wild mammalian species. Such findings should be of particular relevance in emphasizing the need for skilful management of captive wildlife collections, where restricted population size is the norm rather than the exception.

We thank the Government of Tanzania, the Indian Central Government, the Gujarat State Government and particularly Professor K. N. Hirji, Coordinator of the Serengeti Wildlife Research Institute and H. A. Vaishnav, Chief Conservator of Forests (Gujarat) for their generous hospitality and support; and Richard E. Leakey, J. G. Howard, T. Caro, P. P. Raval, B. R. Pandeya, S. Bhuva and the Sakkarbaug Zoo staff for assistance. This study was sponsored by grants from the National Geographic Society, Friends of the National Zoo (FONZ), the US Fish and Wildlife Service and the Smithsonian Institution, Washington, DC.

Received 3 July; accepted 7 August 1987

1. Rice, V. A., Andrews, F. N., Warwick, E. J. \& Legates, J. E. Breeding and Improvement of Farm Animals, 188-190 (McGraw-Hill, New York, 1967).

2. Johannsson, 1. \& Rendel, J. Genetics and Animal Breeding, 257-377 (Oliver and Boyd, Edinburgh, 1968).

3. Wyrobek A. J. Genetics 92, 105-119 (1979).

4. O'Brien, S. J., Wildt, D. E., Goldman, D., Merril, C. R. \& Bush, M. Science 221, 459-462 (1983).

5. O'Brien, S. J., Wildt, D. E. \& Bush, M. Scient. Am. 254, 84-92 (1986),

6. Wildt, D. E. et al. Biol. Reprod. 29, 1019-1025 (1983). 
7. Wildt, D. E. et al Biol Reprod. 36, 351-360 (1987)

8. Myers, N. Int. Wildlife 5, 4-11 (1975).

9. Schaller, G. B. The Serengeti Lion: a Study of Predator-Prey Relations (University of Chicago Press, 1972).

10. Bertram, B. J. Zool. 177, 463-482 (1975)

11. Hanby, J. D. \& Bygott, J. B. in Serengeti: Dynamics of an Ecosystem (eds Sinclair, A. R. E. \& Norton-Griffiths, M.) 249-262 (University of Chicago Press, 1979)

12. Packer, C. in Ecological Aspects of Social Evolution (eds Rubenstein, D. \& Wrangham, F.) 429-451 (Princeton Press, Princeton, 1986).

13. Pusey, A. \& Packer, C. Behaviour (in the press).

14. Joslin, P. in The World's Cats Vol. 1: Ecology and Conservation (ed. Eaton, R. L.) 127-141 (World Wildlife Safari, Winston, Oregon, 1971)

15. Joslin, P. J. Bombay nat. Hist. Soc. 81, 648-664 (1984).

16. Fosbrooke, H. E Afr. Wildlife J. 1, 124-126 (1963).

17. Caldwell, K. J. Soc. Preserv. Faun. Emp. 34, 62-65 (1938)

18. O'Brien, S. J. et al. Natn. Geogr. Res. 3, 114-124 (1987).

19. Ott, R. S. in Current Therapy in Theriogenology (ed. Morrow, D. A.) 125-126 (Saunders, Philadelphia, 1986)

20. Wildt, D. E. in Wild Mammals in Captivity (ed. Lumpkin, S.) (Sinauer, Sunderland, Massachusetts, in the press).

21. Lincoln, G. A. in The Testis (eds Burger, H. \& de Kretser, D.) 255-302 (Raven, New York, 1981)

22. Amann, R. P. in Current Therapy in Theriogenology (ed. Morrow, D. A.) 532-538 (Saunders, Philadelphia, 1986).

23. Bardin, C. W. \& Paulsen, C. A. in Textbook of Endocrinology (ed. Williams, R. H.) 293-354 (Saunders, Philadelphia, 1981).

24. Sharpe, R. M. Biol Reprod. 30, 29-49 (1984).

25. Wildt, D. E., Howard, J. G., Hall, L. L. \& Bush, M. Biol. Reprod. 34, 937-947 (1986)

26. O'Brien, S. J. et al. Science 227, 1428-1434 (1985).

27. Wildt, D. E., Chakraborty, P. K., Meltzer, D. \& Bush, M. Biol. Reprod. 30, 665-672 (1984). 28. Wildt, D. E., Howard, J. G., Chakraborty, P. K. \& Bush, M. Biol. Reprod, 34, 949-959 (1986).

\section{Locomotion of the coelacanth Latimeria chalumnae in its natural environment}

\section{Hans Fricke*, Olaf Reinicke*, Heribert Hofer* \& Werner Nachtigall $\dagger$}

* Max-Planck-Institut für Verhaltensphysiologie,

8131 Seewiesen, FRG

$†$ Zoologisches Institut der Universität des Saariandes, 6600 Saarbrücken, FRG

The coelacanth Latimeria chalumnae is the only living relic of a fossil group of crossopterygian lobe-finned fish ${ }^{1-4}$. We describe observations of its locomotion in a natural environment using six individuals observed from the research submersible Geo in the Indian Ocean at a depth of between 117 and $198 \mathrm{~m}$. Past speculation on the pattern of locomotion has included crawling with the paired fins on the rocky ocean bottom, stalking like a large piscivorous grouper, or even fast swimming in open water ${ }^{4-6}$. Our observations show $L$. chalumnae to be a nocturnal piscivorous drift-hunter, moving very slowly in up- or downwelling currents, while paired fins stabilize and correct the drift motion. Paired and unpaired lobed fins are able to generate thrust. Fast starts are performed with the large caudal fin. Paired fins were not used for locomotion along the bottom. They alternate synchronously in a pattern common in tetrapod locomotion.

L. chalumnae is found near the remote volcanic islands of the Comores in the Western Indian Ocean at a depth of $\sim 200 \mathrm{~m}$. Since their disco, ry in 1938 no observations of free-ranging fish in their natural environment have been made, although locomotory patterns have been observed on line-fished individuals taken to the surface and released in shallow water (refs 7-9, J. L. Geraud, personal communication). These studies indicated a great flexibility of the second dorsal and anal fins which produced some forward thrust by sculling action. The paired fins did not produce swimming movements and the caudal fin did not move at all. The fish remained neutrally buoyant. Line-caught coelacanths are probably under extreme metabolic stress and might suffer decompression sickness after fast surfacing.

We performed 40 dives at 30 different locations around the entire coast of Grande Comore and the north coast of Anjouan and found six fish of $\sim 120-180 \mathrm{~cm}$ body length off a $2-\mathrm{km}$ stretch of the coastline of Grande Comore. These were observed for a
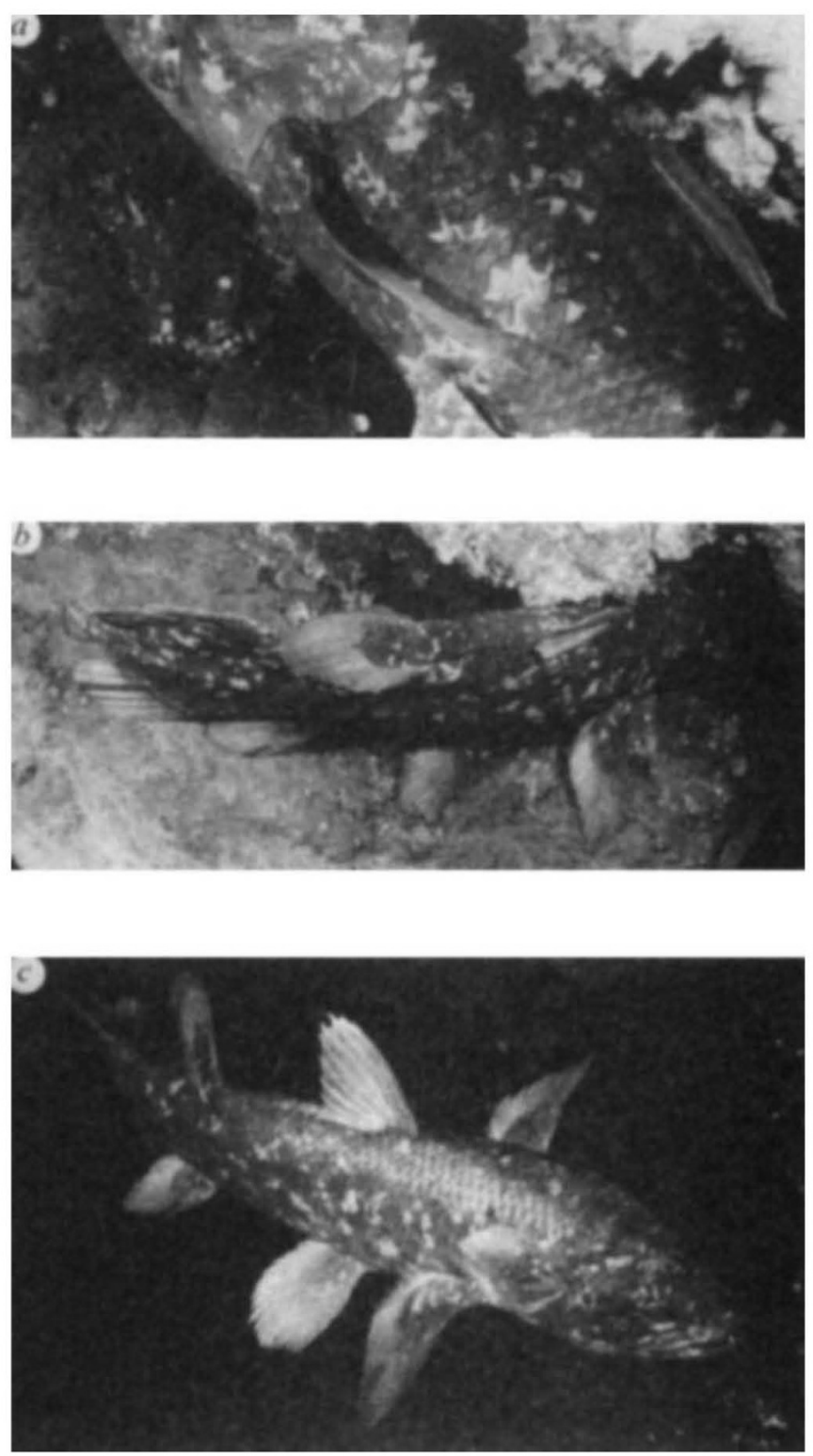

Fig. 1 Contact with the bottom $a$, with pectoral fins folded laterally or $b$, with tips of both paired fins touching the bottom. $c$, Drifting in an upwelling current with paired fins functioning as hydrofoils: they stabilize and correct the 'underwater flying'.

total of $496 \mathrm{~min}$ at night only, between $21: 00$ and $03: 30$, and we have used more than 300 photographs, $90 \mathrm{~min}$ of $16-\mathrm{min}$ cine film and $30 \mathrm{~min}$ of videotape recording for cinematographic analysis. The fish seemed undisturbed by the submersible or by the lights. Two of the six individuals were sitting on the bottom and four were in slow motion. During contact with the bottom, the paired fins were not used for any kind of locomotion. This was confirmed by a six-hour observation of a single individual. It frequently touched the bottom, either with the belly alone, keeping the pectoral fins laterally folded (Fig. 1a), or with the belly and the lobes of the paired fins together (Fig. $1 b$ ); no locomotion was observed.

All individuals took advantage of up- or downwellings and drifted slowly with the current. Both paired fins stabilized and corrected the 'underwater flying' used in a bird wing fashion (Fig. 1c). During drifting, all individuals seemed perfectly oriented in that they avoided obstacles in their environment, apparently detecting them well in advance. Although no feeding 